\title{
Synthesis and characterization of super-hard AlCrTiVZr high-entropy alloy nitride films deposited by HiPIMS
}

\author{
Yi Xu, Guang Li*, Yuan Xia* \\ Institute of Mechanics, Chinese Academy of Sciences, Beijing 100190, PR China \\ Center of Materials Science and Optoelectronics Engineering, University of Chinese Academy of Sciences, Beijing 100049, China
}

\section{A R T I C L E I N F O}

\section{Keywords:}

High entropy alloy (HEA) nitride films

High power impulse magnetron sputtering

(HiPIMS)

Super-hard

$\mathrm{N}_{2}$ gas flow rate

Microstructure

Mechanical properties

\begin{abstract}
A B S T R A C T
Super-hard AlCrTiVZr high-entropy alloy (HEA) nitride films were synthesized by high power impulse magnetron sputtering (HiPIMS) without external heating. The effect of $\mathrm{N}_{2}$ gas low rate $\left(F_{\mathrm{N}}\right)$, ranging from $0 \mathrm{sccm}$ to 20 sccm, on the HiPIMS plasma discharge characterization, element concentration, deposition rate, microstructure, cross-sectional morphology, residual stress, and mechanical properties of films were explored. Results show that increasing $F_{\mathrm{N}}$ increases the HiPIMS discharge current, accompanying with the decreased deposition rate. The saturated nitride (AlCrTiVZr)N films were obtained at $F_{\mathrm{N}}=8 \mathrm{sccm}$ and higher, which exhibit a simple NaCl-type FCC structure. A continuous variation in the microstructure, from amorphous to columnar crystal structure, has been observed for these nitride films. It is discovered that the moderate $F_{\mathrm{N}}$ of $0 \mathrm{sccm}$ to $12 \mathrm{sccm}$ leads to an enhanced bombardment with high-energy particles due to the increased plasma density, while the bombardment effect is weakened because of the decreased plasma energy as the $F_{\mathrm{N}}$ is increased further. The nitride films deposited at $F_{\mathrm{N}}=12 \mathrm{sccm}$ have super-hardness of $41.8 \mathrm{GPa}$ and low wear rate of $2.3 \times 10^{-7} \mathrm{~mm}^{3} / \mathrm{Nm}$. Meanwhile, a change of preferred orientation from $\left(\begin{array}{lll}2 & 0 & 0\end{array}\right)$ to $\left(\begin{array}{lll}1 & 1 & 1\end{array}\right)$ is presented as the $F_{\mathrm{N}}$ increases from 12 sccm to $20 \mathrm{sccm}$.
\end{abstract}

\section{Introduction}

High-entropy alloys (HEAs), which is firstly reported by Cantor et al. and Yeh et al. in $2004[1,2]$, and defined to be formed by five or more principal metal elements with the concentration varying from 5 at.\% to 35 at.\% each $[3,4]$, is a novel alloy design strategy. Due to the combined effects of high mixing entropy, severe lattice distortion and sluggish diffusion [5], complex intermetallic compounds are avoided and single, simple, face-centered cubic (FCC) and/or body-centered cubic (BCC) solid solutions are rather stabilized. Previous researches on HEAs mainly focused on bulk materials due to their extraordinary properties, such as excellent ductility and high strength [6,7], resistance to anneal softening [8], superior resistance to wear [9], oxidation [10] and corrosion [11]. At present, HEA nitride films have begun to be the focus of significant attention in the microelectronic industry thanks to its application in diffusion barriers for $\mathrm{Cu}$ metallization [12]. Besides, its another promising application is to be used as hard films in the cutting tools where high hardness, combined with superior oxidation and corrosion resistance are required [13]. Magnetron sputtering (MS) technology opens an opportunity for us to fabricate HEA nitride films with HEA [14-18]. Nevertheless, due to the low ionization fraction $(<5 \%)$ of sputtered metals in conventional MS $[19,20]$, the deposited films are characterized by a porous and loose columnar structure, which in turn deteriorates the performance of the HEA nitride films.

As a novel ionized physical vapor deposition technology (IPVD), high power impulse magnetron sputtering (HiPIMS) has attracted attention recently [21]. This technology is characterized by high peak power density of several $\mathrm{kW} / \mathrm{cm}^{2}$ at a low duty cycle of less than $10 \%$ and low frequency of less than $10 \mathrm{kHz}$ [22]. With this property, the HiPIMS technique is capable of offering not only highly dense plasma of the order of $10^{13} \mathrm{~cm}^{-3}$ but also broadened energy distribution function (IEDF) of the sputtered particles, which has been found to allow for an intense bombardment of high-energy particle and an increased adatom mobility on the growing films $[23,24]$. Hence, the HiPIMS-deposited films exhibit higher density, improving interface adhesion and lower surface roughness. Solovyev et al. [25] have investigated the $\mathrm{Cu}$ films deposited using direct current magnetron sputtering (DCMS) and HiPIMS. Compared to films deposited by DCMS, the HiPIMS-deposited films have smoother surface and well-defined denser nanocrystalline structure. Lattermann et al. [26] have reported on highly dense, low roughness and droplet-free TiN films deposited by HiPIMS without

\footnotetext{
* Corresponding authors at: Institute of Mechanics, Chinese Academy of Sciences, Beijing 100190, PR China.

E-mail addresses: xuyi@imech.ac.cn (Y.Xu), lghit@imech.ac.cn (G. Li), xia@imech.ac.cn (Y. Xia).
} 
additional heating or substrate bias voltage, and revealed how an intense energetic ion irradiation by $\mathrm{Ti}^{+}, \mathrm{Ti}^{2+}$ and $\mathrm{N}^{+}$ions provided the desired densification of the TiN films. Ma et al. [27] have synthesized the TiAlSiN nanocomposite films deposited by HiPIMS at various bias voltages, and reported a denser microstructure, a lower surface roughness and a significantly higher hardness than for DCMS-deposited films. According to these publications, the HiPIMS, compared with the conventional MS, is a promising technique to strengthen the control of plasma environment, the microstructure as well as the properties of deposited films. Therefore, the synthesis and characterization of the HiPIMS-deposited HEA nitride films are worthy of further exploration and expected to present more excellent properties.

The main objective of this study is therefore to assess the feasibility of HiPIMS in synthesizing HEA nitride films. The HEA target composed with five strong nitride forming elements, $\mathrm{Al}, \mathrm{Cr}, \mathrm{Ti}, \mathrm{V}, \mathrm{Zr}$, was designed to deposit nitride films. The $\mathrm{N}_{2}$ gas flow rate, known as a vital factor determining film properties, was tuned to investigate corresponding changes in the plasma discharge characteristic, element composition, microstructure, morphology, hardness and tribological properties.

\section{Experiment details}

\subsection{Film deposition}

All depositions of AlCrTiVZr nitride films by HiPIMS were carried out in an automated multipurpose deposition apparatus with a base pressure of below $3 \times 10^{-3} \mathrm{~Pa}$. Detailed information of this deposition system have already been mentioned in other publications [27]. As indicated in Fig. 1, the HEA mosaic target, with $76 \mathrm{~mm}$ in diameter and $3 \mathrm{~mm}$ in thickness, was composed of sectors of $\mathrm{AlCr}$, TiZr and $\mathrm{V}$, and these sectors were all fixed on the $\mathrm{Cu}$ base target. Sputtering yields of $\mathrm{AlCr}$, TiZr and $\mathrm{V}$ were separately calculated with TRIM software in order to determine the relative surface fraction of each sector required in fabricating a HEA mosaic target for the deposition of stoichiometric AlCrTiVZr of equimolar composition. The angles of the AlCr, TiZr and V sector were $48^{\circ}, 90^{\circ}$ and $42^{\circ}$, respectively. A pulse power generator (HighPulse 4002, Huttinger Electronic) was applied to power the HEA target. Films were deposited on cemented carbide (WC-10 wt $\%$ Co), which prior to deposition were cleaned ultrasonically in deionized water, ethanol, and dried in air blow. A thin silicon p-type (lllll 00 ) wafer of thickness $300 \pm 10 \mu \mathrm{m}$ was also used as a substrate to accurately estimate the residual stress.

The synthesis of AlCrTiVZr nitride films was conducted in an atmosphere of high purity $\operatorname{Ar}(99.999 \%)$ gas and $\mathrm{N}_{2}$ (99.999\%) gas. Prior to deposition, samples were further processed by $20 \mathrm{~min} \mathrm{Ar}^{+}$sputteretching at $2 \mathrm{~Pa} \mathrm{Ar}$ pressure and $-800 \mathrm{~V}$ substrate bias voltage in order to remove surface contaminants and oxidation layer. During the deposition of nitride films, a fixed Ar gas flow rate of $150 \mathrm{sccm}$ was introduced into the vacuum chamber, while the $\mathrm{N}_{2}$ gas flow rate (denoted as $F_{\mathrm{N}}$ ) was selected as the controlling parameter, which was set as 0 $\mathrm{sccm}, 4 \mathrm{sccm}, 8 \mathrm{sccm}, 12 \mathrm{sccm}, 16 \mathrm{sccm}$ and $20 \mathrm{sccm}$, corresponding to the Sample H1, H2, H3, H4, H5 and H6, respectively. The target and

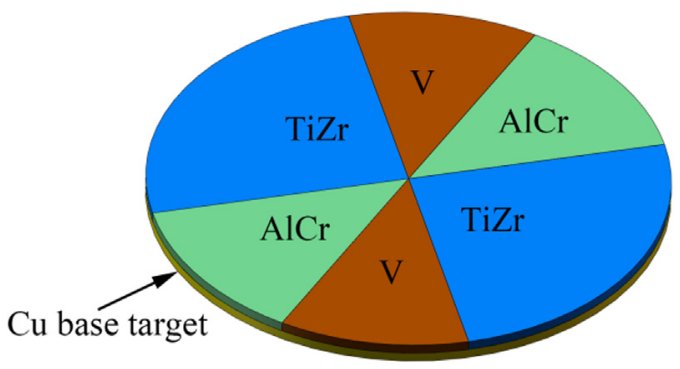

AlCr sector : $48^{\circ} \quad$ TiZr sector : $90^{\circ} \quad \mathrm{V}$ sector : $42^{\circ}$

Fig. 1. Schematic diagram of AlCrTiVZr Mosaic target.
Table 1

Details of deposition parameters.

\begin{tabular}{ll} 
Parameter & Value \\
\cline { 2 - 2 } & Sample H1/H2/H3/H4/H5/H6 \\
\hline Target-substrate Distance (mm) & 75 \\
Ar flow rate (sccm) & 150 \\
$\mathrm{~N}_{2}$ gas flow rate (sccm) & $0 / 4 / 8 / 12 / 16 / 20$ \\
Deposition Pressure (Pa) & $0.4 / 0.43 / 0.47 / 0.5 / 0.52 / 0.54$ \\
Deposition Time (min) & 60 \\
Substrate Bias Voltage (V) & -75 \\
Pulse Frequency (Hz) & 200 \\
Pulse Duration ( $\mu$ s) & 100 \\
Pulse Negative Voltage (V) & -560 \\
Peak Discharge Current (A) & $52 / 67 / 76 / 84 / 92 / 101$ \\
\end{tabular}

samples were placed together face to face. No external heating was applied during the deposition process. The detailed deposition parameters of these nitride films were summarized in Table 1.

\subsection{Characterization}

Current-voltage-time characteristics of the HiPIMS discharge were measured by a high voltage probe (Tektronix, IHVP250) and a current monitor (PEM, CWT1B), respectively and recorded with a digital oscilloscope (Tektronix, TDS1002B). The element composition of the AlCrTiVZr nitride films was determined with an Electron Probe Micro Analysis (EPMA, JEOL, JAX8230) and at least three measurements were implemented for each sample. Films thicknesses and cross-sectional morphology were observed under a field emission scanning electron microscope (FE-SEM, ZEISS, SUPRA55). The crystallographic structure of films was characterized by a glancing incident angel X-ray diffraction (GIXRD, Rigaku, D/MAX 2500) with $\mathrm{Cu} \mathrm{K} \alpha$ radiation at the incident angle of $1^{\circ}$. The average grain size of the films was further calculated from the full-width at half-maximum by Scherrer's equation. Hardness and modulus were investigated by means of a nanoindentation tester (Agilent, Nano Indenter G200) with a load precision of $50 \mathrm{nN}$ using continuous stiffness mode (CSM). A constant indentation depth of $300 \mathrm{~nm}$ (less than one-tenth of the films thickness) was chosen so as to eliminate the substrate effects. The residual stress $\sigma$ of the films was determined by substrate-curvature method and calculated using the Stoney equation [28]. The tribological behaviors of the films were obtained by a ball-on-disk reciprocating friction and wear equipment (MFT-R4000) at a relative humidity of approximately $22 \% \mathrm{RH}$ and temperature of $23 \pm 1{ }^{\circ} \mathrm{C}$. GCr15 bearing steel balls with a diameter of $5 \mathrm{~mm}$ were used against the films at $240 \mathrm{~mm} / \mathrm{min}$ sliding velocity and a load of $30 \mathrm{~N}$. The wear track length and sliding time were $5 \mathrm{~mm}$ and $120 \mathrm{~min}$, respectively. After the wear experiments, the wear rates $\left(\mathrm{mm}^{3} / \mathrm{Nm}\right)$ were further calculated from the cross-sectional areas of the wear tracks measured by a laser confocal scanning microscope (Olympus, Ols4000).

\section{Results and discussion}

\subsection{HiPIMS plasma discharge characterization}

Fig. 2 displays the HiPIMS discharge current waveforms at various $F_{\mathrm{N}}$, where the discharge voltage is fixed to $-560 \mathrm{~V}$. Since the shape and the level of the discharge voltages are constant in all depositions, only the voltage waveform at $F_{\mathrm{N}}=4 \mathrm{sccm}$ is plotted as reference. As can be seen in Fig. 2, the discharge current increases rapidly to its peak value after the onset of the pulse, and then it decreases slowly as a result of gas rarefaction effect [29]. For a pure Ar discharge, the discharge peak current is $52 \mathrm{~A}$. With the introduction of $\mathrm{N}_{2}$ gas into the gas atmosphere, discharge peak current obviously exhibits an increasing tendency and increases sharply to $101 \mathrm{~A}$ at $F_{\mathrm{N}}=20 \mathrm{sccm}$. For HiPIMS 


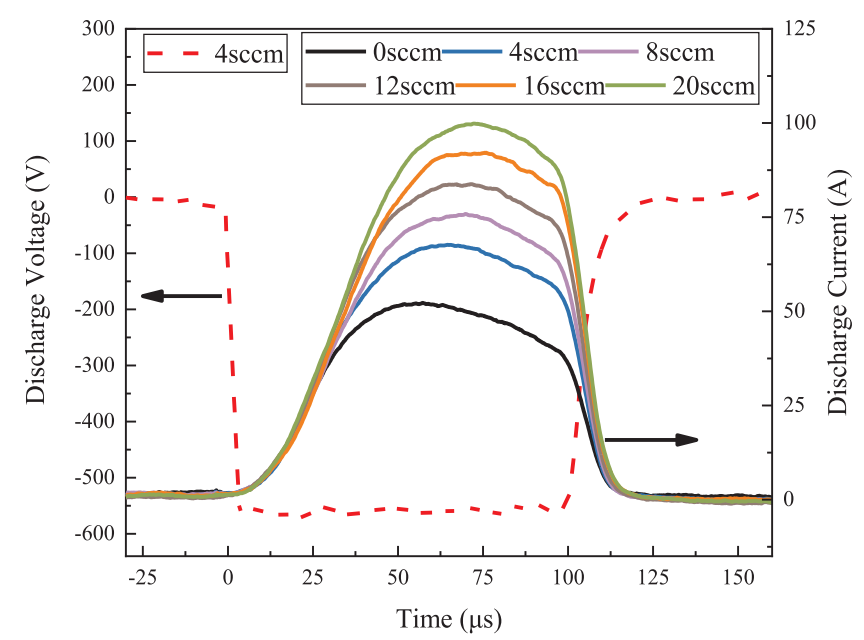

Fig. 2. The HiPIMS discharge current waveforms at various $F_{\mathrm{N}}$ and a voltage waveform at $F_{\mathrm{N}}=4 \mathrm{sccm}$ in a pulsed period.

plasma characteristics, a larger discharge current is a macroscopic expression of the increase of high-energy particles, which also means an increase in plasma density and high ion-to-neutral ratio. As illustrated in Table 1 , the deposition pressure increases with the increasing of $F_{\mathrm{N}}$. Therefore, the observed increase in discharge current is attributed to an increase in collision frequencies between sputtered or atmosphere particles and energetic electrons. On the other hand, $\mathrm{N}$ and $\mathrm{N}_{2}$ are more apt to be ionized than Ar due to the higher ionization potential for $\mathrm{Ar}$ [30]. Then a higher atomic ions $\mathrm{N}^{1+}$ fraction originated from the dissociation of the energetic $\mathrm{N}_{2}{ }^{+}$molecule upon impact on the target will be produced in the target vicinity [31]. Hence, these large fluxes of $\mathrm{N}^{1+}$ to the cathode will bring about a significant increase in the discharge current carried by ions.

\subsection{Chemical composition and deposition rate}

The chemical composition of the AlCrTiVZr nitride films deposited at various $F_{\mathrm{N}}$ are plotted in Fig. 3(a). It is important to note that although the relative concentrations of $\mathrm{Al}$ and $\mathrm{Cr}$ are slightly higher than that of $\mathrm{Ti}, \mathrm{V}$ and $\mathrm{Zr}$ in both HEA and its nitride films, the relative concentration of each element still meets the design criteria of HEAs. The difference in relative concentrations of elements can be explained by the fact that the etching depth of AlCr sector is larger than that of other regions with the increase of deposition time due to the higher sputtering yields of AlCr sector. As a result, the magnetic strength of the

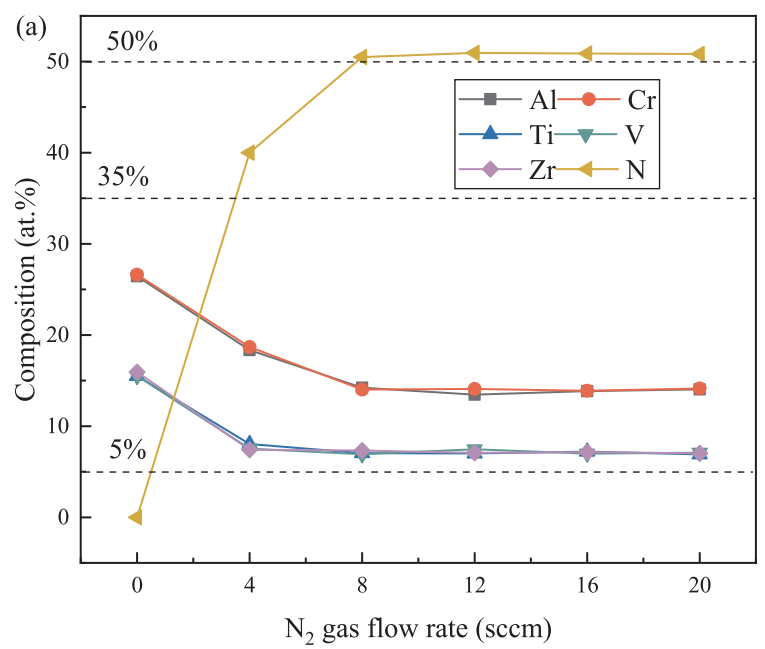

AlCr sector becomes higher. These results provide valuable reference and guidance for the design optimization of HEA mosaic target in the following researches. Not surprisingly, with the addition of $\mathrm{N}_{2}$ gas, the relative concentration of $\mathrm{N}$ in the deposited films dramatically increases, and then approaches about $50 \%$ at $F_{\mathrm{N}}=8 \mathrm{sccm}$. This is reasonable since all target elements have a strong affinity for $\mathrm{N}$ atoms. Then to further increase the $F_{\mathrm{N}}$ from $8 \mathrm{sccm}$ to $20 \mathrm{sccm}$, little variation is observed in the relative concentration of each element. For Sample $\mathrm{H} 3, \mathrm{H} 4, \mathrm{H} 5$ and H6, they can be regarded as saturated nitrides and expressed as the stoichiometric form of (AlCrTiVZr)N films.

Fig. 3(b) presents the deposition rates of AlCrTiVZr nitride films deposited at various $F_{\mathrm{N}}$. It can be seen that the deposition rate decreases from $67.8 \mathrm{~nm} / \mathrm{min}$ to $57.3 \mathrm{~nm} / \mathrm{min}$ with $F_{\mathrm{N}}$ increasing from $0 \mathrm{sccm}$ to $20 \mathrm{sccm}$. Such variations in the deposition rate with varied $F_{\mathrm{N}}$ are mainly due to the formation of a nitride layer on the target surface, which in turn reduces the quantity of sputtered particles from the target, thereby resulting in the occurrence of so-called "target poisoning". In addition, unlike $\mathrm{Ar}^{+}$, the sputtering yield of $\mathrm{N}_{2}$ gas ion is lower due to the lower mass of $\mathrm{N}$ [32], which is another key factor contributing to the decrease in the deposition rate.

\subsection{Microstructures and morphology}

The GIXRD patterns of AlCrTiVZr nitride films deposited at various $F_{\mathrm{N}}$ are depicted in Fig. 4. For HEA films, only one broad peak representing the amorphous structure is found to exist. Theoretically, film deposition can be considered to be an extremely rapid quenching process, in which case, deposition atoms have insufficient kinetics to form preferred crystalline structures [33]. On the other hand, the complete mutual solubility of different elements is enhanced by high mixing entropy [1]. The severe lattice distortion caused by the incorporation of atoms with different size leads to topological instability and also decreases the driving force for the transformation from amorphous to crystalline configuration. Meanwhile, sluggish diffusion effect [1], originating from the difficulty for different atoms to have cooperative diffusion, can slow the crystallization kinetics. Therefore, an amorphization phenomenon is presented for the AlCrTiVZr HEA films. With the $F_{\mathrm{N}}$ increasing to $4 \mathrm{sccm}$, it is observed that the AlCrTiVZr nitride films still demonstrate an amorphous structure, which may be attributed to the lower $\mathrm{N}$ reactive concentration (39 at. \%) in films. With the $F_{\mathrm{N}}$ further increasing to $8 \mathrm{sccm}$ or higher, only one set of peaks representing FCC NaCl-Type structure can be observed in each pattern, which indicates that these nitride films contain a simple solid solution nitride phase rather than separate nitrides coexisting with one another. This phenomenon can also be explained by complete mutual solubility

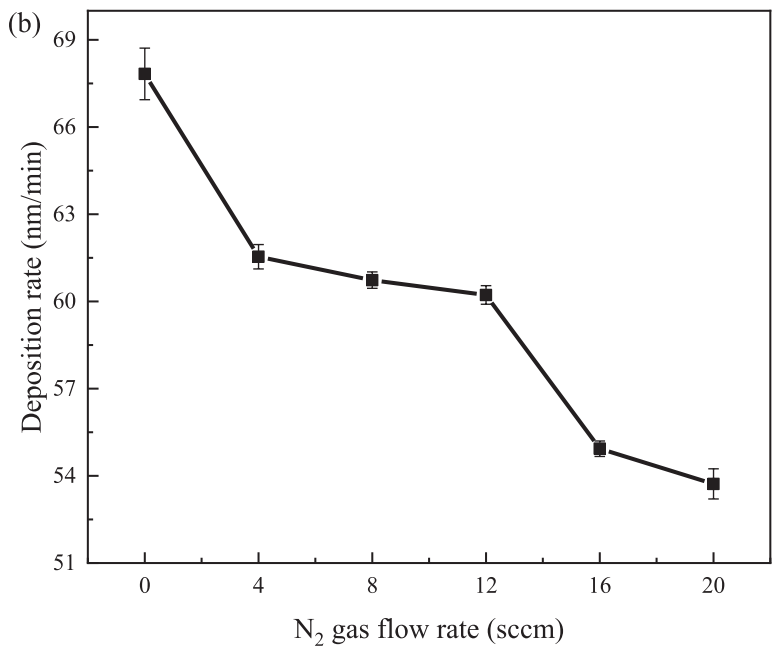

Fig. 3. Chemical composition (a) and deposition rates (b) of the AlCrTiVZr nitride films as a function of the $F_{\mathrm{N}}$. 


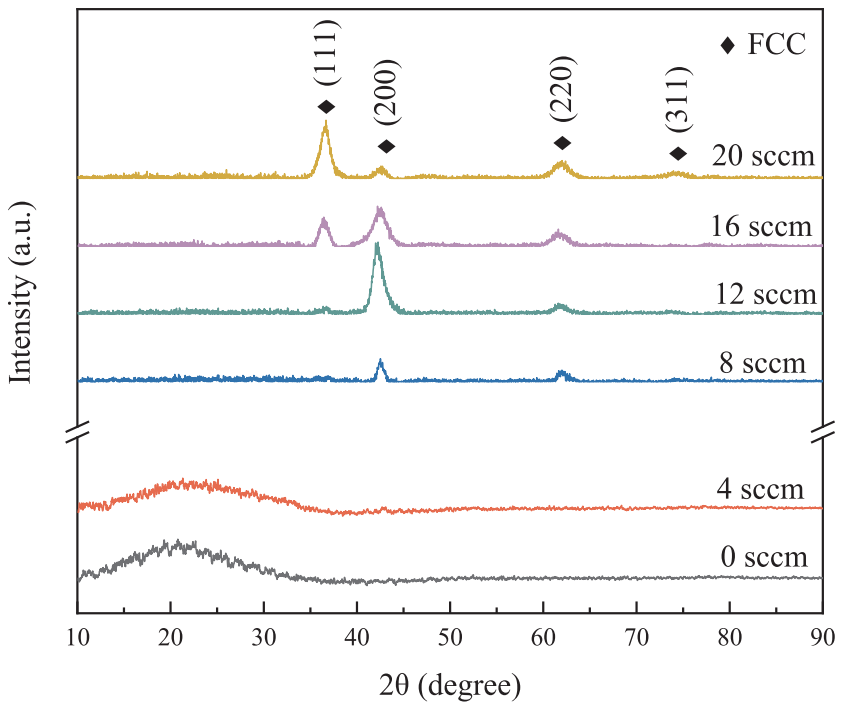

Fig. 4. X-ray diffraction patterns of the AlCrTiVZr nitride films deposited at various $F_{\mathrm{N}}$.

mentioned above, and thus different elements can compromise with each other to produce a simple FCC solid solution structure. Moreover, in the current HEA system, although the preferred AlN structure is hexagonal close-packed (HCP) structure, $\mathrm{CrN}$, TiN, VN and $\mathrm{ZrN}$ all have FCC structures $[15,34]$. Therefore, it is reasonable to conclude that the FCC structure dominates the crystal structure of (AlCrTiVZr)N films.

In addition, it can be observed that with the $F_{\mathrm{N}}$ increasing from 8 $\mathrm{sccm}$ to $12 \mathrm{sccm}$, the $\left(\begin{array}{lll}2 & 0 & 0\end{array}\right)$ peak is dramatically intensified. For the growth of the NaCl-Type FCC nitride film, the competition between strain and surface energy determines the preferred orientation [35]. The plane ( $\left.\begin{array}{lll}1 & 1 & 1\end{array}\right)$ has the lowest strain energy, while the plane $\left(\begin{array}{lll}2 & 0 & 0\end{array}\right)$ has the lowest surface energy [35]. As more high-energy particles will be generated with the increase of $F_{\mathrm{N}}$, the bombardment between deposition flux and the growing film will be enhanced, which can make a positive impact on the adatom mobility, thereby promoting the growth of lowest surface energy planes. In such situation, an increase in $\left(\begin{array}{lll}2 & 0 & 0\end{array}\right)$ peak intensity is as expected. When the $F_{\mathrm{N}}$ increases to $16 \mathrm{sccm}$, the $\left(\begin{array}{lll}2 & 0 & 0\end{array}\right)$ peak intensity decreases gradually, consistent with an obvious increase in the (llll 1111$)$ peak intensity, while $\left(\begin{array}{lll}2 & 0 & 0\end{array}\right)$ peak is still dominant. Nevertheless, increasing the $F_{\mathrm{N}}$ further up to $20 \mathrm{sccm}$ results in a shift of the preferred orientation from $\left(\begin{array}{lll}2 & 0 & 0\end{array}\right)$ to $\left(\begin{array}{lll}1 & 1 & 1\end{array}\right)$. Such variation of preferred orientation can be attributed to the fact that decreased plasma energy dominates the growth of films a higher $F_{\mathrm{N}}$. With the increase of $F_{\mathrm{N}}$, the mean free path of the deposition particle becomes smaller as a result of the increasing deposition pressure [36]. Therefore, the deposition particles will collide with Ar atoms or $\mathrm{N}_{2}$ molecules more frequently in the path, causing the deposition particles to be scattered and deflected from their paths to the substrate, thereby reducing plasma energy as well as the energy to the surface of films. For deposition particles with low energy, films prefer a $\left(\begin{array}{lll}1 & 1 & 1\end{array}\right)$ orientation for the highest number of atoms per unit area can be incorporated at low energy sites [24].

Meanwhile, the average grain sizes and lattice constants of the (AlCrTiVZr)N films deposited at various $F_{\mathrm{N}}$ are shown in Fig. 5 . It can be seen that the grain size decreases from $12.8 \mathrm{~nm}$ to $6.3 \mathrm{~nm}$ as the $F_{\mathrm{N}}$ is raised from $8 \mathrm{sccm}$ to $12 \mathrm{sccm}$. For $F_{\mathrm{N}}$ that is greater than $12 \mathrm{sccm}$, the grain size increases slightly. However, the lattice constant changes in an opposite manner. As mentioned above, the increase in the $F_{\mathrm{N}}$ leads to not only more bombardment between deposition flux and the growing films, but also a reduction in the plasma energy. Therefore, the initial decrease in grain size is probably a consequence of the enhanced bombardment effect due to the increasing plasma density. The bombardment effect can produce more defects which reduce the energy

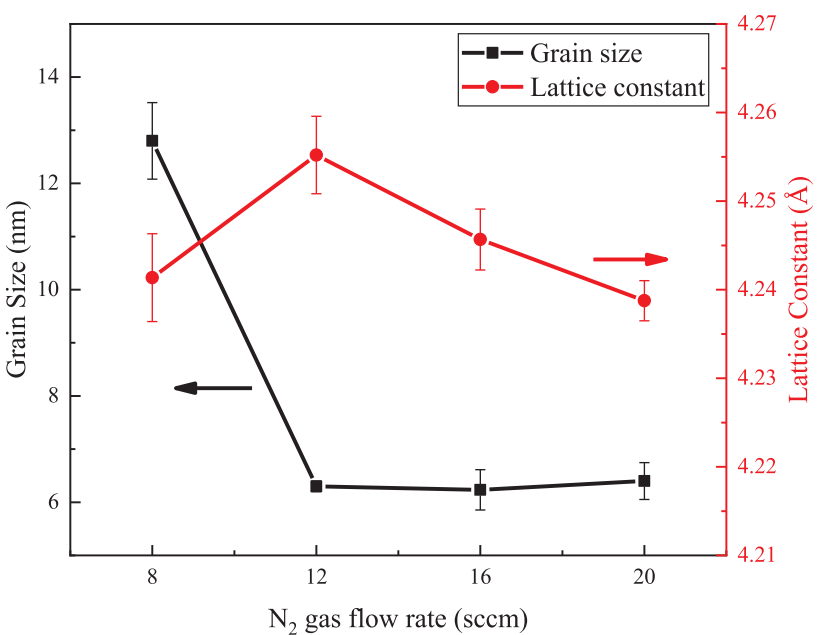

Fig. 5. The average grain sizes and lattice constants of (AlCrTiVZr)N films deposited at $F_{\mathrm{N}}$ of $8 \mathrm{sccm}, 12 \mathrm{sccm}, 16 \mathrm{sccm}$ and $20 \mathrm{sccm}$.

needed for nucleation and make nucleation more competitive relative to grain growth [37]. For lattice constant, the initial increase with the increasing $F_{\mathrm{N}}$ is due to the excessive addition of $\mathrm{N}$ atoms and point defects into the films $[38,39]$, which is induced by enhanced bombardment effect. However, at a higher $F_{\mathrm{N}}$, the reduction in the plasma energy will weaken the bombardment of high-energy particles, which is beneficial to the increase of the grain size and the decrease of lattice constant.

The cross-sectional morphologies of AlCrTiVZr nitride films deposited at various $F_{\mathrm{N}}$ are illustrated in Fig. 6 . The HEA films show a very dense and smooth cross-section structure in which no pores or voids are observed. When the $F_{\mathrm{N}}$ increases to $4 \mathrm{sccm}$, the cross-section structure still remains unchanged, as shown in Fig. 6(b). However, at $F_{\mathrm{N}}=8$ $\mathrm{sccm}$, a columnar crystal structure emerges. By further increasing $F_{\mathrm{N}}$ to $12 \mathrm{sccm}$ or higher, the columnar crystal structure is still visible, and the films present looser structure with more defined columnar. The weakened bombardment effect and decreased adatoms mobility resulted from decreased plasma energy may be the main reason for the appearance of columnar structure. Although the columnar crystal structure can be found in (AlCrTiVZr)N films deposited by HiPIMS, both the length and the diameter of the columns are smaller as compared to the films deposited by conventional MS [34]. This difference results from the deposition flux with high ion-to-neutral in HiPIMS process, which promotes the adatoms mobility on the surface of the growing films and significantly reduces the intra- and inter-columnar porosity.

Moreover, it is worth noting that the structure of the (AlCrTiVZr)N films obviously shows two layers separated by a boundary. As shown in Fig. 6(c)-(f), these boundaries are marked by a yellow dash line in each sample. The columnar crystal structure is seen in the upper layer, while a dense and smooth structure without visible grain feature is observed in the lower layer. Meanwhile, the thickness of the lower layer decreases with the increase of $F_{\mathrm{N}}$. In order to determine the structure of these (AlCrTiVZr)N films deposited in the initial deposition stage, GIXRD was used to further characterize the (AlCrTiVZr)N films with a deposition time of $30 \mathrm{~min}$. As shown in Fig. 7, only one large broad peak can be observed in the four patterns, which indicates that the lower layer has an amorphous structure rather than FCC NaCl-Type structure. There are several factors accounting for the initial growth of (AlCrTiVZr)N films on cemented carbide substrates: First, as mentioned above, the growth of (AlCrTiVZr)N films is significantly dependent on the combined effects of high mixing entropy, severe lattice distortion and sluggish diffusion, which contributes to the formation of the amorphous nitrides. Second, according to the GIXRD results (see Fig. 4), the lattice constant of (AlCrTiVZr)N films is in the range of $4.23 \AA$ $-4.25 \AA$, while the lattice constant of cemented carbide mainly made 


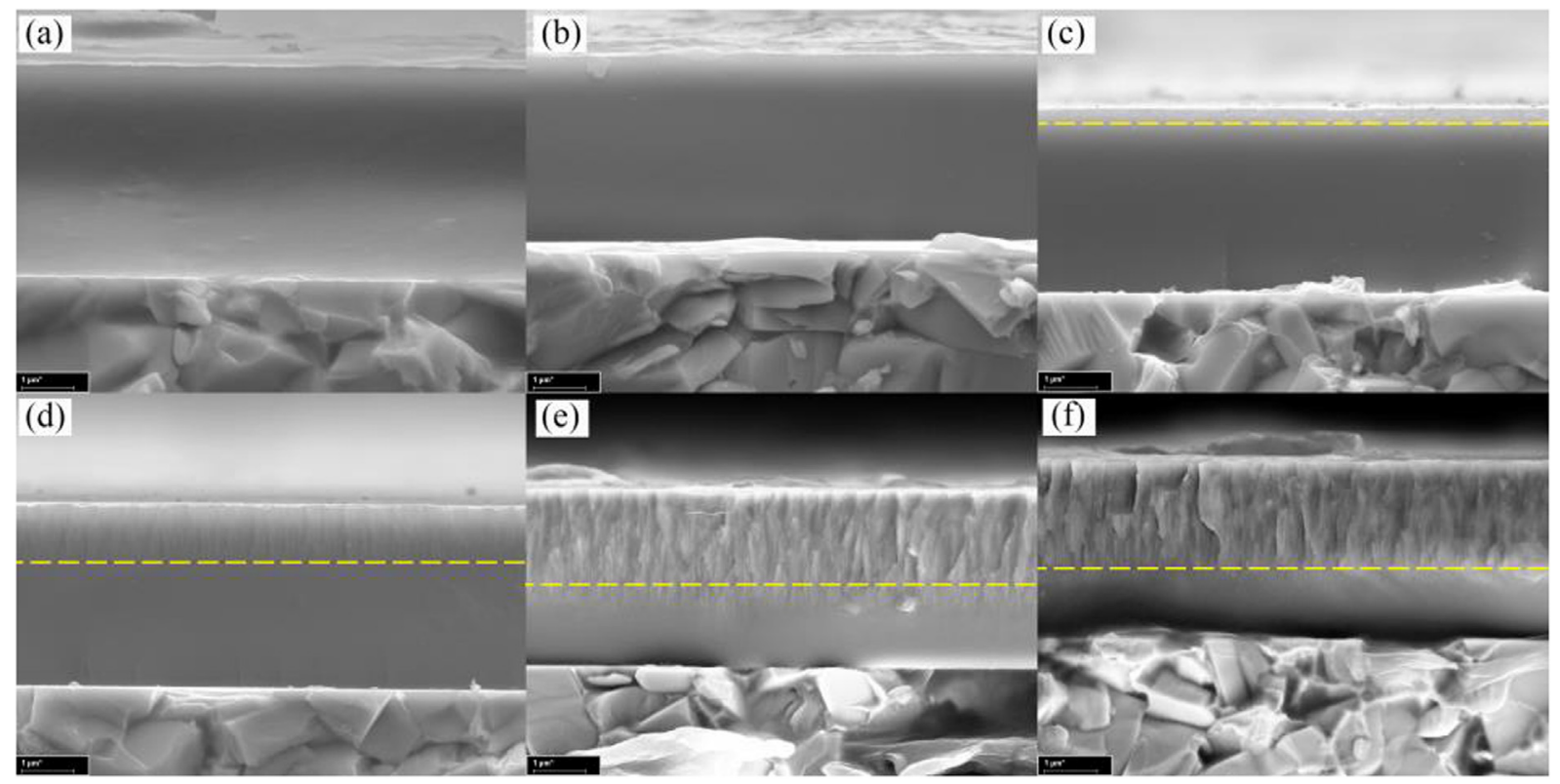

Fig. 6. FE-SEM cross-section micrographs of the AlCrTiVZr nitride films deposited at $F_{\mathrm{N}}$ of: (a) $0 \mathrm{sccm}$, (b) $4 \mathrm{sccm}$, (c) $8 \mathrm{sccm}$, (d) $12 \mathrm{sccm}$, (e) $16 \mathrm{sccm}$, (f) $20 \mathrm{sccm}$. The deposition time is $60 \mathrm{~min}$.

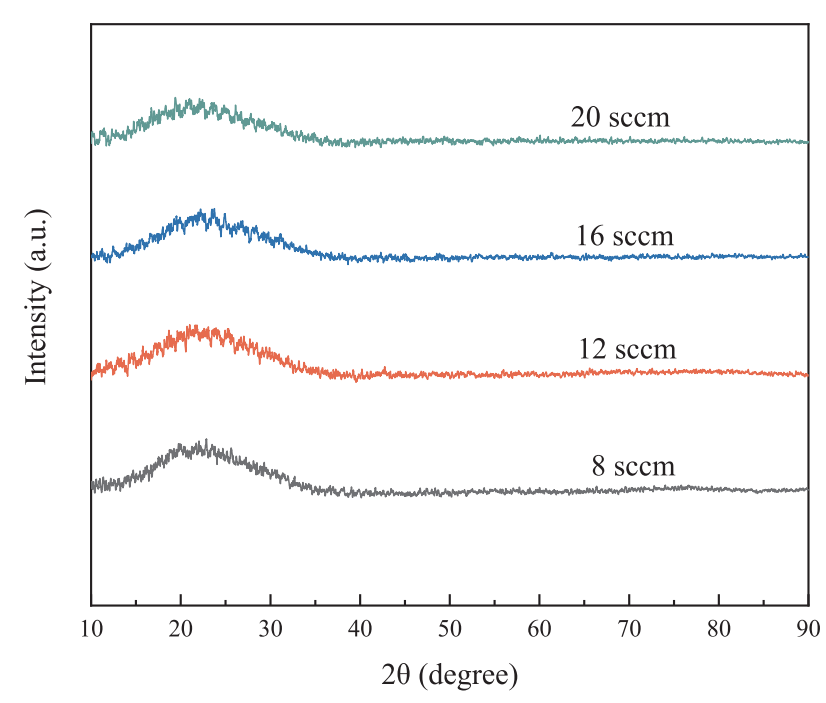

Fig. 7. X-ray diffraction patterns of the (AlCrTiVZr)N films deposited at various $\mathrm{N}_{2}$ gas flow rates. The deposition time is $30 \mathrm{~min}$.

of tungsten carbide with HCP structure is $\mathrm{a}=\mathrm{b}=2.906 \AA$, $c=2.838 \AA$. Thus, the (AlCrTiVZr)N films suffer huge stress in initial films deposition process due to the large lattice mismatch between (AlCrTiVZr)N films and substrate. Third, intense bombardment of highenergy particles caused by HiPIMS on the growing film is expected, which can positively affect the efficient atomic rearrangement and formation of larger residual stress, as shown in Fig. 8. Considering all the factors discussed above, the amorphization of the crystalline phase is motivated. With the increase of thickness, namely, to stay away from the interface between (AlCrTiVZr)N films and the substrates, the residual stress is relieved. Accordingly, the lattice order starts to form nano grains and the columnar crystal structure with an FCC crystal phase begins to develop. Furthermore, with the increased $F_{N}$, the bombardment effect is weakened as a result of decreased plasma energy, which promotes the formation of crystalline phase, thereby resulting in a decrease in the thickness of the amorphous layer.

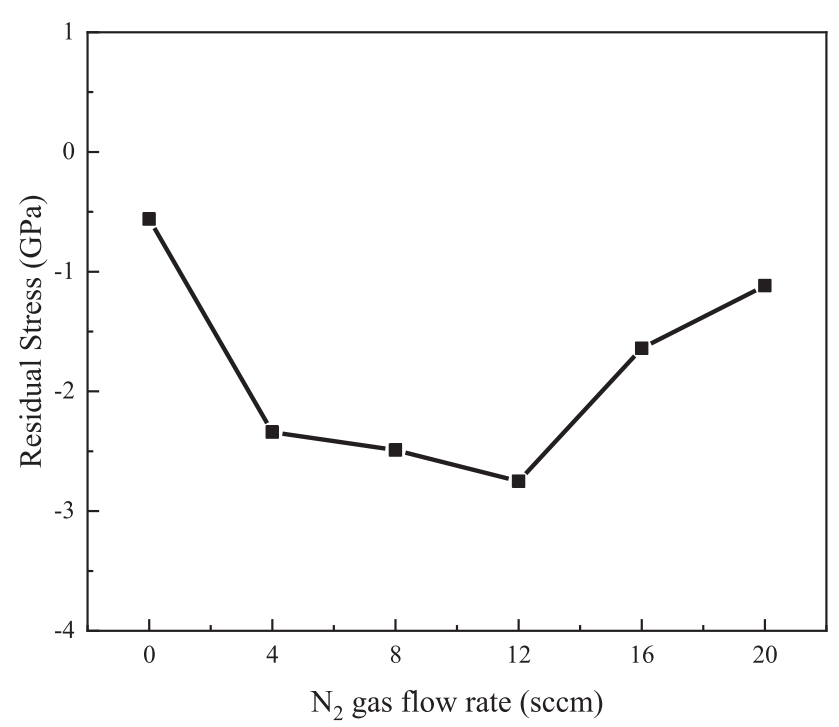

Fig. 8. Residual stress of the AlCrTiVZr nitride films as a function of the $F_{\mathrm{N}}$.

\subsection{Residual stress}

As shown in Fig. 8, the AlCrTiVZr nitride films all possess a compressive residual stress that initially increases as the $F_{\mathrm{N}}$ increases, and then gradually decreases from a peak of $-2.75 \mathrm{GPa}$ at $F_{\mathrm{N}}=12 \mathrm{sccm}$ to $-1.11 \mathrm{GPa}$ at $F_{\mathrm{N}}=20 \mathrm{sccm}$. Generally speaking, the measured residual stress of the films mainly consists of two parts: thermal stress and intrinsic stress. Since no additional heating system was applied during the deposition process, it can be inferred that the residual stress basically comes from intrinsic stress, which originates from the bombardment of high-energy particles on the growing film surface. Therefore, the initial increase in the residual stress is due to the increased bombardment effect. The high-energy bombardment particles, including atmosphere and sputtered metal ions, will implant the growing films and generate more defects, which will lead to the expansion of lattice, thereby inducing formation of compressive stress [40]. Moreover, the decrease in residual stress, under the situation that the $F_{N}$ is further increased, is attributed to the weakened bombardment of high-energy particles 


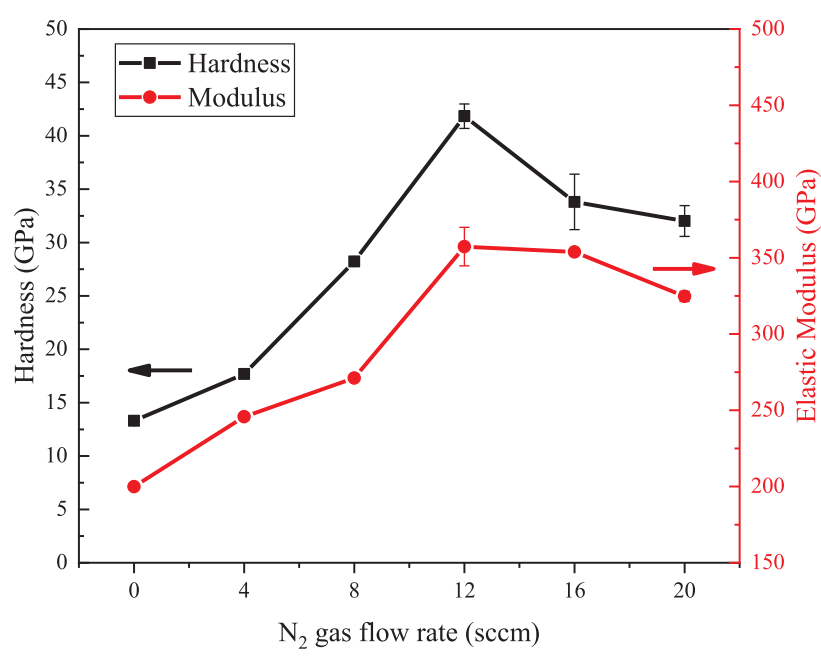

Fig. 9. Hardness and modulus of the AlCrTiVZr nitride films as a function of the $F_{\mathrm{N}}$.

mentioned above.

\subsection{Hardness and modulus}

The hardness and elastic modulus of the AlCrTiVZr nitride films deposited at various $F_{\mathrm{N}}$ are depicted in Fig. 9. For HEA films, the hardness and elastic modulus are measured as $13.3 \mathrm{GPa}$ and $199 \mathrm{GPa}$, respectively. With the $F_{\mathrm{N}}$ increasing to $8 \mathrm{sccm}$, both the hardness and elastic modulus sharply increase to $28 \mathrm{GPa}$ and $271 \mathrm{GPa}$, respectively. The enhanced hardness can be explained by the formation of a nanocrystalline nitride phase as well as the strong covalent bonding between $\mathrm{N}$ and target elements. By further increasing the $F_{\mathrm{N}}$ to $12 \mathrm{sccm}$, the hardness and elastic modulus reach their maximum values of $41 \mathrm{GPa}$ and $363 \mathrm{GPa}$, respectively. For $F_{\mathrm{N}}$ greater than $12 \mathrm{sccm}$, the hardness and elastic modulus present a decreasing tendency. Furthermore, the HiPIMS-deposited (AlCrTiVZr)N films have a much higher hardness than that deposited by conventional magnetron sputtering, which exhibit hardness values of 4.5-11 GPa [34].

According to above measurements, it can be concluded that the $F_{\mathrm{N}}$ has a significant influence on the preferred orientation, grain size, densification, and residual stress of the films, which in turn, determines the hardness of the (AlCrTiVZr)N films. In general, increased hardness is observed in the FCC nitride films with more pronounced $\left(\begin{array}{lll}1 & 1 & 1\end{array}\right)$ orientation, which is the hardest orientation resulted from the geometrical strengthening [41]. However, as shown in Fig. 4, the Sample H4 $(12 \mathrm{sccm})$ with a preferred $\left(\begin{array}{lll}2 & 0 & 0\end{array}\right)$ orientation exhibits the highest hardness. Meanwhile, by the Hall-Petch effect [42], the smaller grain size produces more remarkable strengthening effect as more grain boundaries act as impediments to the dislocation motion and resulting slip. However, when the grain size is less than $10 \mathrm{~nm}$, the sliding of grain boundary occurs and thus causes a softening effect, namely inverse Hall-Petch effect. As shown in Fig. 5, the Sample H3, and Sample H4, H5 and H6 are located Hall-Petch and inverse Hall-Petch regions, respectively, and there is no obvious difference among different (AlCrTiVZr)N films in terms of the grain size. Hence, preferred orientation and grain size are insufficient to explain the variation of hardness. The residual stress, as discussed in Section 3.4, resulting from the incorporation of defects will hinder plastic flow during the deformation and then increase the hardness [16]. In this study, the variation of the residual stress is well consistent with that of the film's hardness. Therefore, the residual stress should be responsible for the variation in the measured hardness. In addition, from the SEM observations, the microstructure evolution from densely packed structure to loose columnar structure is acquired, which produces more poles at grain and column boundaries, thereby weakening the effect of boundary strengthening. This loose microstructure is another important factor contributing to the decrease in the measured hardness from Sample H4 (12 sccm) to H6 $(20 \mathrm{sccm})$.

\subsection{Tribological properties}

Fig. 10(a) presents the friction coefficient evolution of the (AlCrTiVZr)N films deposited at various $F_{\mathrm{N}}$ with the sliding time. The friction coefficient curves for all samples show similar feature that increases sharply in the initial stage, and then reach to a relatively steady state quickly. The average friction coefficient is calculated after about $5 \mathrm{~min}$ of the sliding time, as shown in the inserted figure of Fig. 10(a). It seems that the $F_{\mathrm{N}}$ has little effect on the friction coefficient of these (AlCrTiVZr)N films and all average friction coefficients remain around 0.5. Fig. 10(b) further shows the wear rates of the (AlCrTiVZr)N films deposited at various $F_{\mathrm{N}}$. The wear rate of the (AlCrTiVZr)N films initially decreases as the $F_{\mathrm{N}}$ increases, and reaches to the minimum value of about $2.38 \times 10^{-7} \mathrm{~mm}^{3} / \mathrm{Nm}$ at $F_{\mathrm{N}}=12 \mathrm{sccm}$. The decrease in wear rate is primarily due to the contribution of the increased films' hardness. By further increasing $F_{\mathrm{N}}$ to $20 \mathrm{sccm}$, wear rate increases to about $9.19 \times 10^{-7} \mathrm{~mm}^{3} / \mathrm{Nm}$. Such variation can be partly attributed to a decrease in the hardness and in part to the formation of looser with
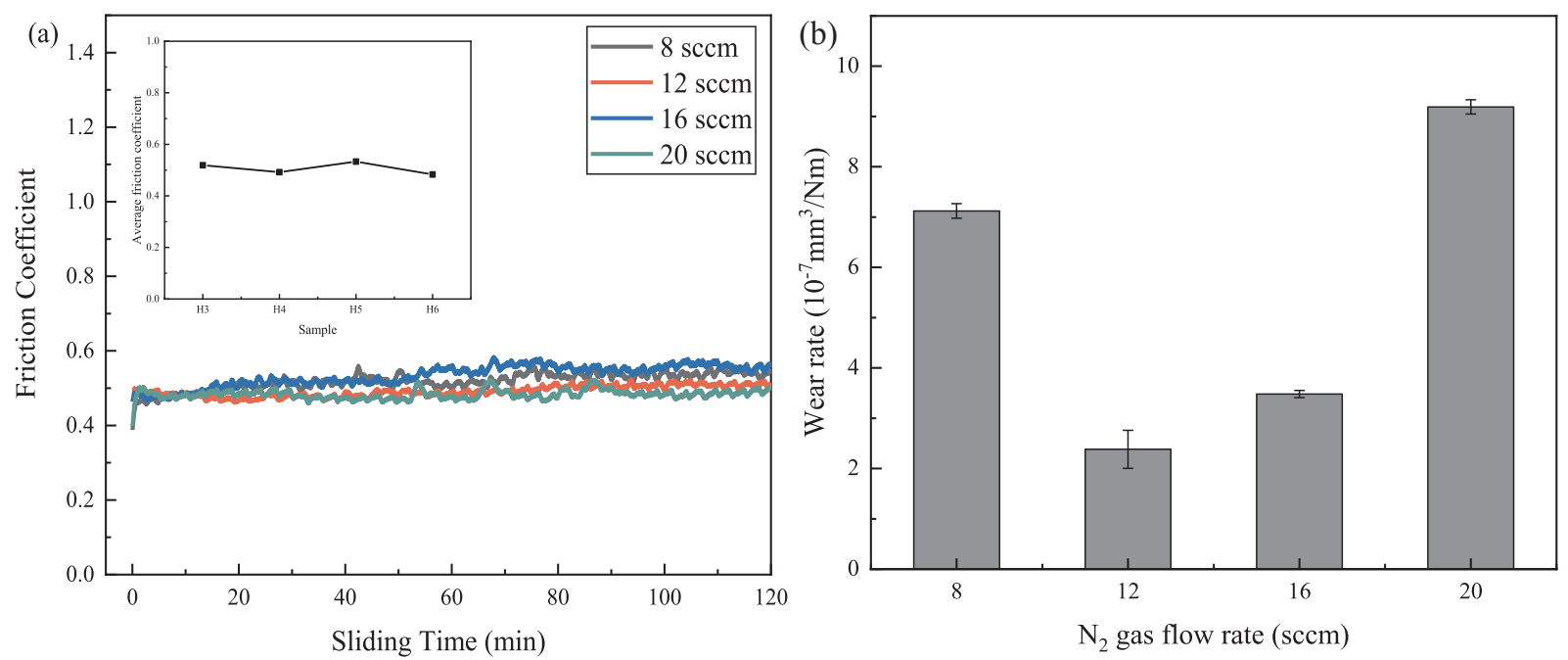

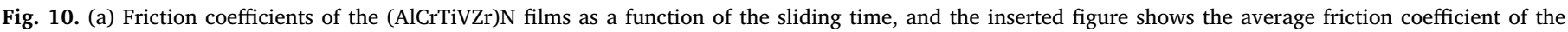
(AlCrTiVZr)N films as a function of the $F_{\mathrm{N}}$. (b) Wear rates of the (AlCrTiVZr)N films as a function of the $F_{\mathrm{N}}$. 
more defined columnar structure. Moreover, in contrast to other HEA nitride films deposited by conventional MS, which have a wear rate of the order of $10^{-6} \mathrm{~mm}^{3} / \mathrm{Nm}$ [28,43-45], the HiPIMS-deposited (AlCrTiVZr)N films show more excellent wear resistance.

The above study results have demonstrated that HiPIMS is an effective way to improve microstructure and properties by controlling plasma environment for AlCrTiVZr HEA nitride films. It can be found that a moderate increase in $F_{N}$ can significantly enhances the bombardment of high-energy particles to the growing films due to the increased plasma density, which results in an increase in (2 00 ) peak intensity, a decrease in grain size and an increase in residual stress. As a result, the films deposited at $F_{N}=12 \mathrm{sccm}$ exhibit a super-hardness and low wear rate, which are mainly attributed to a high residual stress. However, at high $F_{N}$, the bombardment effect is weakened due to the decreased plasm energy, which leads to a decrease in residual stress and looser structure with more defined columnar, thereby deteriorating the performance of the HEA nitride films.

\section{Conclusions}

In this study, AlCrTiVZr nitride films were synthesized by HiPIMS at various $F_{\mathrm{N}}$ without additional heating. The peak discharge current dramatically increases from 52 A to 101 A while the growth rate decreases from $67.8 \mathrm{~nm} / \mathrm{min}$ to $57.3 \mathrm{~nm} / \mathrm{min}$ with structure of the films becoming loose. The nitride films reach a saturated state at $F_{\mathrm{N}}=8 \mathrm{sccm}$ or higher. According to the XRD observations, both the AlCrTiVZr films and the unsaturated nitride films exhibit an amorphous structure, while the (AlCrTiVZr)N films present a simple FCC solid-solution phase. Moreover, an amorphous layer is found in the initial deposition process of (AlCrTiVZr)N films, which results from high mixing entropy, mismatch in the lattice between films and substrate, and bombardment of high-energy particles. On the other hand, the $F_{\mathrm{N}}$ of $12 \mathrm{sccm}$ is a critical value for the growth of the films. As the $F_{\mathrm{N}}$ increases from $0 \mathrm{sccm}$ to 12 sccm, the enhanced bombardment of high-energy particles due to the increased plasma density results in an increase in $(200)$ peak intensity, a decrease of grain size to $6.3 \mathrm{~nm}$, an increase of residual stress to $-2.75 \mathrm{GPa}$. At $F_{\mathrm{N}}=12 \mathrm{sccm}$, the films have super-hardness of 41.8 $\mathrm{GPa}$ and low wear rate of $2.3 \times 10^{-7} \mathrm{~mm}^{3} / \mathrm{Nm}$. When the $F_{\mathrm{N}}$ increases over the critical value, the bombardment of high-energy particles is weakened due to the decreased plasma energy, thereby resulting in a shift of the preferred orientation from $\left(\begin{array}{lll}2 & 0 & 0\end{array}\right)$ to $\left(\begin{array}{lll}1 & 1 & 1\end{array}\right)$ and a contrary tendency in the properties of films.

\section{CRediT authorship contribution statement}

Yi Xu: Methodology, Formal analysis, Investigation, Writing - original draft. Guang Li: Validation, Resources, Supervision. Yuan Xia: Conceptualization, Writing - review \& editing, Project administration, Funding acquisition.

\section{Declaration of Competing Interest}

The authors declare that they have no known competing financial interests or personal relationships that could have appeared to influence the work reported in this paper.

\section{Acknowledgements}

This works were jointly supported by National Nature Science Foundation of China (Nos. 51871230 \& 51701229) and the Strategic Priority Research Program of the Chinese Academy of Sciences (No. XDB22040503).

\section{References}

[1] J.W. Yeh, S.K. Chen, S.J. Lin, J.Y. Gan, T.S. Chin, T.T. Shun, C.H. Tsau, S.Y. Chang, Nanostructured High-Entropy Alloys with Multiple Principal Elements: Novel Alloy Design Concepts and Outcomes, Adv. Eng. Mater. 6 (2004) 299-303.

[2] B. Cantor, I.T.H. Chang, P. Knight, A.J.B. Vincent, Microstructural development in equiatomic multicomponent alloys, Mater. Sci. Eng., A 375-377 (2004) 213-218.

[3] Y. Zhang, T.T. Zuo, Z. Tang, M.C. Gao, K.A. Dahmen, P.K. Liaw, Z.P. Lu, Microstructures and properties of high-entropy alloys, Prog. Mater. Sci. 61 (2014) 1-93.

[4] M.H. Tsai, J.W. Yeh, High-Entropy Alloys: A Critical Review, Mater. Res. Lett. 2 (2014) 107-123.

[5] J. Yeh, Alloy Design Strategies and Future Trends in High-Entropy Alloys, JOM-US 65 (2013) 1759-1771.

[6] H. Yao, Z. Tan, D. He, Z. Zhou, Z. Zhou, Y. Xue, L. Cui, L. Chen, G. Wang, Y. Yang, High strength and ductility AlCrFeNiV high entropy alloy with hierarchically heterogeneous microstructure prepared by selective laser melting, J. Alloy. Compd 813 (2020) 152196

[7] Q. Ding, Y. Zhang, X. Chen, X. Fu, D. Chen, S. Chen, L. Gu, F. Wei, H. Bei, Y. Gao, M. Wen, J. Li, Z. Zhang, T. Zhu, R.O. Ritchie, Q. Yu, Tuning element distribution, structure and properties by composition in high-entropy alloys, Nature 574 (2019) 223-227.

[8] W. Zhang, P. Liaw, Y. Zhang, A Novel Low-Activation VCrFeTaxWx ( $\mathrm{x}=0.1,0.2$, 0.3, 0.4, and 1) High-Entropy Alloys with Excellent Heat-Softening Resistance, Entropy-Switz 20 (2018) 951, https://doi.org/10.3390/e20120951.

[9] R.B. Nair, H.S. Arora, A.V. Boyana, P. Saiteja, H.S. Grewal, Tribological behavior of microwave synthesized high entropy alloy claddings, Wear 436-437 (2019) 203028.

[10] Y. Cai, L. Zhu, Y. Cui, K. Geng, S. Marwana Manladan, Z. Luo, High-temperature oxidation behavior of FeCoCrNiAlx high-entropy alloy coatings, Mater. Res. Express 6 (2019) 126552

[11] S. Zheng, Z. Cai, J. Pu, C. Zeng, S. Chen, R. Chen, L. Wang, A feasible method for the fabrication of VAlTiCrSi amorphous high entropy alloy film with outstanding anticorrosion property, Appl. Surf. Sci. 483 (2019) 870-874.

[12] S. Chang, M. Chen, High thermal stability of AlCrTaTiZr nitride film as diffusion barrier for copper metallization, Thin Solid Films 517 (2009) 4961-4965.

[13] C. Chang, C. Yang, C. Sung, C. Hsu, Structure and tribological behavior of (AlCrNbSiTiV)N film deposited using direct current magnetron sputtering and high power impulse magnetron sputtering, Thin Solid Films 668 (2018) 63-68.

[14] P. Huang, J. Yeh, Effects of nitrogen content on structure and mechanical properties of multi-element (AlCrNbSiTiV)N coating, Surf. Coat. Technol. 203 (2009) 1891-1896.

[15] S. Liang, D. Tsai, Z. Chang, H. Sung, Y. Lin, Y. Yeh, M. Deng, F. Shieu, Structural and mechanical properties of multi-element (TiVCrZrHf)N coatings by reactive magnetron sputtering, Appl. Surf. Sci. 258 (2011) 399-403.

[16] M. Hsieh, M. Tsai, W. Shen, J. Yeh, Structure and properties of two Al-Cr-Nb-Si-Ti high-entropy nitride coatings, Surf. Coat. Technol. 221 (2013) 118-123.

[17] T.K. Chen, T.T. Shun, J.W. Yeh, M.S. Wong, Nanostructured nitride films of multielement high-entropy alloys by reactive DC sputtering, Surf. Coat. Technol. 188-189 (2004) 193-200.

[18] D. Tsai, Y. Huang, S. Lin, S. Liang, F. Shieu, Effect of nitrogen flow ratios on the structure and mechanical properties of (TiVCrZrY)N coatings prepared by reactive magnetron sputtering, Appl. Surf. Sci. 257 (2010) 1361-1367.

[19] S. Konstantinidis, A. Ricard, M. Ganciu, J.P. Dauchot, C. Ranea, M. Hecq, Measurement of ionic and neutral densities in amplified magnetron discharges by pulsed absorption spectroscopy, J. Appl. Phys. 95 (2004) 2900-2905.

[20] C. Christou, Z.H. Barber, Ionization of sputtered material in a planar magnetron discharge, J. Vacuum Sci. Technol. A: Vacuum, Surfaces, Films 18 (2000) 2897-2907.

[21] V. Kouznetsov, K. Macák, J.M. Schneider, U. Helmersson, I. Petrov, A novel pulsed magnetron sputter technique utilizing very high target power densities, Surf. Coat. Technol. 122 (1999) 290-293.

[22] K. Sarakinos, J. Alami, S. Konstantinidis, High power pulsed magnetron sputtering: A review on scientific and engineering state of the art, Surf. Coat. Technol. 204 (2010) 1661-1684.

[23] A.P. Ehiasarian, A. Vetushka, Y.A. Gonzalvo, G. Sáfrán, L. Székely, P.B. Barna, Influence of high power impulse magnetron sputtering plasma ionization on the microstructure of TiN thin films, J. Appl. Phys. 109 (2011) 104314.

[24] Z. Wang, D. Zhang, P. Ke, X. Liu, A. Wang, Influence of Substrate Negative Bias on Structure and Properties of TiN Coatings Prepared by Hybrid HIPIMS Method, J. Mater. Sci. Technol. 31 (2015) 37-42.

[25] A.A. Solovyev, V.O. Oskirko, V.A. Semenov, K.V. Oskomov, S.V. Rabotkin, Comparative Study of Cu Films Prepared by DC, High-Power Pulsed and Burst Magnetron Sputtering, J. Electron. Mater. 45 (2016) 4052-4060.

[26] M. Lattemann, U. Helmersson, J.E. Greene, Fully dense, non-faceted 111-textured high power impulse magnetron sputtering TiN films grown in the absence of substrate heating and bias, Thin Solid Films 518 (2010) 5978-5980.

[27] Q. Ma, L. Li, Y. Xu, J. Gu, L. Wang, Y. Xu, Effect of bias voltage on TiAlSiN nanocomposite coatings deposited by HiPIMS, Appl. Surf. Sci. 392 (2017) 826-833.

[28] K. Cheng, C. Lai, S. Lin, J. Yeh, Structural and mechanical properties of multi-element (AlCrMoTaTiZr)Nx coatings by reactive magnetron sputtering, Thin Solid Films 519 (2011) 3185-3190.

[29] J. Alami, K. Sarakinos, G. Mark, M. Wuttig, On the deposition rate in a high power pulsed magnetron sputtering discharge, Appl. Phys. Lett. 89 (2006) 154104.

[30] F. Magnus, O.B. Sveinsson, S. Olafsson, J.T. Gudmundsson, Current-voltage-time 
characteristics of the reactive Ar/N2 high power impulse magnetron sputtering discharge, J. Appl. Phys. 110 (2011) 83306.

[31] E. Oks, A. Anders, Evolution of the plasma composition of a high power impulse magnetron sputtering system studied with a time-of-flight spectrometer, J. Appl. Phys. 105 (2009) 93304

[32] C. Lai, S. Lin, J. Yeh, S. Chang, Preparation and characterization of AlCrTaTiZ multi-element nitride coatings, Surf. Coat. Technol. 201 (2006) 3275-3280.

[33] C. Tsai, S. Lai, K. Cheng, M. Tsai, A. Davison, C. Tsau, J. Yeh, Strong amorphization of high-entropy AlBCrSiTi nitride film, Thin Solid Films 520 (2012) 2613-2618.

[34] Z. Chang, S. Liang, S. Han, Y. Chen, F. Shieu, Characteristics of TiVCrAlZr multielement nitride films prepared by reactive sputtering, Nucl. Instrum. Methods Phys. Res., Sect. B 268 (2010) 2504-2509.

[35] J. Pelleg, L.Z. Zevin, S. Lungo, Reactive-sputter-deposited TiN films on glass substrates, Thin Solid Films 197 (1991) 1991.

[36] Y. Xu, L. Li, S. Luo, Q. Lu, J. Gu, N. Lei, C. Huo, Self-enhanced plasma discharge effect in the deposition of diamond-like carbon films on the inner surface of slender tube, Appl. Surf. Sci. 393 (2017) 467-473.

[37] G. Håkansson, J.-E. Sundgren, D. McIntyre, J.E. Greene, W.-D. Münz, Microstructure and physical properties of polycrystalline metastable Ti0.5Al0.5N alloys grown by d.c. magnetron sputter deposition, Thin Solid Films 153 (1-3)
(1987) 55-65, https://doi.org/10.1016/0040-6090(87)90169-6.

[38] J.H. Kang, K.J. Kim, Structural, optical, and electronic properties of cubic TiNx compounds, J. Appl. Phys. 86 (1999) 346-350.

[39] S.J. Bull, A.M. Jones, A.R. McCabe, Residual stress in ion-assisted coatings, Surf Coat. Tech. 54-55 (1992) 173-179.

[40] M.M.M. Bilek, D.R. McKenzie, A comprehensive model of stress generation and relief processes in thin films deposited with energetic ions, Surf. Coat. Technol. 200 (2006) 4345-4354.

[41] D. Tsai, Z. Chang, B. Kuo, M. Shiao, S. Chang, F. Shieu, Structural morphology and characterization of (AlCrMoTaTi)N coating deposited via magnetron sputtering, Appl. Surf. Sci. 282 (2013) 789-797.

[42] S. Vepřek, The search for novel, superhard materials, J. Vacuum Sci. Technol. A: Vacuum, Surfaces, Films 17 (1999) 2401-2420.

[43] X. Feng, K. Zhang, Y. Zheng, H. Zhou, Z. Wan, Effect of Zr content on structure and mechanical properties of (CrTaNbMoV)Zrx high-entropy alloy films, Nucl. Instrum. Methods Phys. Res., Sect. B 457 (2019) 56-62.

[44] C. Lai, K. Cheng, S. Lin, J. Yeh, Mechanical and tribological properties of multielement (AlCrTaTiZr)N coatings, Surf. Coat. Technol. 202 (2008) 3732-3738.

[45] K. Cheng, C. Weng, C. Lai, S. Lin, Study on adhesion and wear resistance of multielement (AlCrTaTiZr)N coatings, Thin Solid Films 517 (2009) 4989-4993. 